\title{
Power Adaptation for Cognitive Radio Systems Under an Average SINR Loss Constraint in the Absence of Path Loss Information
}

\author{
Berkan Dulek • Sinan Gezici • Ryo Sawai • \\ Ryota Kimura
}

Published online: 9 November 2013

C Springer Science+Business Media New York 2013

\begin{abstract}
An upper bound is derived on the capacity of a cognitive radio system by considering the effects of path loss and log-normal shadowing simultaneously for a single-cell network. Assuming that the cognitive radio is informed only of the shadow fading between the secondary (cognitive) transmitter and primary receiver, the capacity is achieved via the water-filling power allocation strategy under an average primary signal to secondary interference plus noise ratio loss constraint. Contrary to the perfect channel state information requirement at the secondary system (SS), the transmit power control of the SS is accomplished in the absence of any path loss estimates. For this purpose, a method for estimating the instantaneous value of the shadow fading is also presented. A detailed analysis of the proposed power adaptation strategy is conducted through various numerical simulations.
\end{abstract}

Keywords Cognitive radio $\cdot$ Capacity $\cdot$ Water-filling $\cdot$ Power control $\cdot$ Shadowing · Path loss

\section{Introduction}

As pointed out by the Federal Communications Commission (FCC), the main cause of spectrum scarcity is the inefficient spectrum allocation policy [1]. Majority of the frequency bands are devoted to specific users with exclusive licenses, and stringent limits are imposed on their maximum transmitted power levels to prevent mutual interference over all times. With the

B. Dulek · S. Gezici $(\bowtie)$

Department of Electrical and Electronics Engineering, Bilkent University, 06800 Bilkent, Ankara, Turkey e-mail: gezici@ee.bilkent.edu.tr

B. Dulek

e-mail:dulek@ee.bilkent.edu.tr

R. Sawai · R. Kimura

Sony Corporation, Gotenyama Technology Center, Tokyo 141-0022, Japan

e-mail: Ryo.Sawai@jp.sony.com

R. Kimura

e-mail: Ryota.Kimura@jp.sony.com 
advent of technology, sophisticated transmitters with adaptable parameters are manufactured and receivers become more immune to inter-system interference. This process has brought the necessity to adapt new spectrum usage policies over already allocated frequency bands to enhance the performance of current systems and make room for new technologies.

Mitola introduced the concept of cognitive radio (CR) which relies on permitting secondary system (SS) users to utilize the resources devoted to the primary system (PS) opportunistically [2]. The current approaches can be grouped under two categories: (a) Opportunistic spectrum access, and (b) Spectrum sharing [3]. In the first approach, an SS user tries to detect the absence of PS users and makes use of the spectrum holes. On the other hand, the second approach allows an SS user to operate simultaneously with PS users under the condition that the interference from the SS user should not compromise the reception quality of the licensed PS users. In the latter scenario, it is crucial to adjust the transmit power and rate of the SS user in an adaptive manner to maximize the throughput while restricting the interference to PS users. More explicitly, the SS user may utilize the channel more efficiently (with higher power) when the path between the secondary system transmitter and primary system receiver is subject to deep fading. As a result, ergodic (Shannon) and outage capacities of cognitive radio are studied extensively in the literature under different fading environments (Rayleigh, Hoyt, Rice, Nakagami-m, Log-normal, etc). Several performance metrics have been proposed to optimize secondary spectrum utilization, including but not limited to peak/average interference at the PS receiver, minimum outage capacity of PS, maximum transmission outage probability of PS, peak/average transmit power of SS and bandwidth available to SS. First, we present an outline of the results obtained so far in this field.

\subsection{Prior Work}

In [4], the capacity for the SS user is derived for different types of single-user and multiuser AWGN channels under constraints on the average received power at the PS user (a.k.a. the interference temperature (IT) constraint). In the absence of fading, it is shown that a solution similar to the achievable rate under channel inversion based power adaptation policy can be obtained for point-to-point AWGN channels. The similarity of the solution to the transmit power-constrained case is evident since the received power is a deterministic multiple of the transmitted power for a non-fading AWGN channel. The discussion is also extended to network cases including relay networks, multiple access channels with dependent sources and feedback, and collaborative communications scenarios. The case for time-varying PS and SS channels due to fading is investigated in [5] by employing the methods already introduced in [6-8] about the capacity of fading channels under various transmit power constraints. The ergodic capacity of the SS is evaluated in the case of perfect channel state information (CSI) and interference constraints at the PS user's receiver for different fading scenarios. Contrary to the case when the transmit power is constrained, it is found out that channel capacity in severe fading conditions (e.g., Rayleigh or log-normal fading) exceeds that of the non fading AWGN channel. This result is attributed to the fact that the SS user may utilize the channel more efficiently (with higher power) when the path between SS transmitter and PS receiver is subject to deep fading. By considering average and peak interference power constraints, the authors derive the optimal power allocation schemes which turn out to be time-varying versions of the water-filling algorithm. In [9], the authors derive the outage capacity with its optimum power allocation policy for Rayleigh flat-fading channel under both average and peak received power constraints at the PS receiver. In [10], more power constraints related to the transmit power limitation of the SS user are incorporated in addition to the interference 
power constraint at the PS receiver, and the corresponding optimal power allocation strategies are studied to achieve the ergodic capacity and the outage capacity of the SS user under block fading (BF) channel conditions. A capacity increase is noted for the case of average over peak transmission/interference power constraints.

In [11], an information-theoretic analysis is presented to characterize the optimal transmission strategy and the corresponding channel capacity for an SS user operating under both transmit and interference power constraints imposed at a set of PS receivers. It is shown that by employing multi-antennas at the secondary transmitter, significant capacity improvements can be attained even under stringent power constraints. In [12], single-input multiple-output multiple access channels (SIMO-MAC) are considered under interference constraints at the PS users and individual peak transmit power constraints at the SS users. In [13], an upper bound on the capacity for a cognitive user is derived by prohibiting any cooperation between primary and secondary users. It is shown that the capacity under average and peak secondary to primary interference to signal ratio (ISR) constraints can be achieved via the water-filling power allocation strategy when all the links are subject to identical and independent Rayleigh fading. In addition to channel fading, a simplified path loss model is employed to incorporate effects due to network geometry in a more realistic scenario. Finally, the case of multiple primary receivers is addressed and it is demonstrated numerically that the capacity of cognitive radio grows with triple-log scaling if opportunistic transmission scheduling is employed inside the PS.

Various attempts have been made to replace IT constraint at the PS receiver with more advanced techniques to enhance PS and SS performances. In [14], the authors suggest the use of minimum-PS-outage-capacity requirement instead of the IT constraint to adjust the SS transmission. Despite its improved performance, this new constraint requires additional knowledge about the PS CSI at the SS transmitter. To reduce the operational complexity of the cognitive user, this novel minimum outage capacity requirement for the PS is converted into an approximate interference power constraint that has to be satisfied by the SS user in [15]. In [16], the achievable transmission rate of the SS user is maximized without inflicting any outage capacity loss at the PS via opportunistically adapting the transmit power. Another novel constraint that utilizes the additional CSI of the PS fading channel is proposed to replace the IT condition in [17]. In addition to the average/peak transmit power constraints, the maximum transmission outage probability of the PS user is limited to stay below a desired target value. As a result, excess interference from the SS user can be accommodated by exploiting the non-zero outage probability margin. The corresponding optimal power allocation strategies of the SS are determined to maximize its ergodic and outage capacity. It is reported that significant capacity gains can be obtained for the SS user with respect to the conventional IT constraint under the same PS user outage probability. In [18], a cognitive radio network is considered where multiple SS users benefit from the spectrum of the PS under fading channels via the frequency division multiple access scheme. A total bandwidth constraint is introduced in addition to the peak/average transmit power constraints at the SS users and the peak/average IT constraints at the PS receiver. Closed-form solutions for optimal bandwidth allocation are determined for any given power allocation.

\subsection{Our Contribution}

Most of the prior analysis in this subject focuses on fading channels while paying comparatively less attention to the effect of network geometry on the capacity of cognitive user, mainly due to inherent analytical difficulties associated with the latter [13]. In practice, it is of utmost importance to take into account the relative distances between respective nodes in 
a communications network since path loss constitutes the most important determinant of the achievable rates.

In the following analysis, we derive an upper bound on the ergodic capacity of the cognitive radio by considering detailed interference scenarios due to network geometry in addition to log-normal shadowing for a single-cell network system. Assuming that the secondary (cognitive) user is only informed of the shadow fading between secondary transmitter and primary receiver, a closed form expression is obtained for the SS power transmission strategy under an average primary signal to secondary interference plus noise ratio (SINR) loss constraint. Contrary to perfect CSI requirement at the SS transmitter, the transmission power control of the SS can be accomplished in the absence of any path loss estimates. To that aim, a method to estimate the instantaneous value of the shadow fading is also given.

The remainder of the paper is organized as follows. In Sect. 2, we state the system model and assumptions for the analysis of the single-cell network. Then, secondary system transmission power control problem is studied under an average SINR loss constraint in the absence of any path loss estimates in Sect. 3. Section 4 discusses the approaches on how to estimate the instantaneous value of the shadow fading, which is the sole determinant of the proposed strategy. Next, we conduct a number of numerical simulations to obtain an in-depth analysis of the suggested power adaptation method in Sect. 5. Concluding remarks are made in Sect. 6.

\section{System Model}

For the PS model, a single-cell environment is assumed in our analysis as shown in Fig. 1. The inter-node distance state vector is denoted by $\mathbf{r}=\left[r_{s s}, r_{s p}, r_{p s}, r_{p p}\right]$, where subscript $s$ and $p$ denote primary and secondary, respectively. The first subscript indicates the transmitter

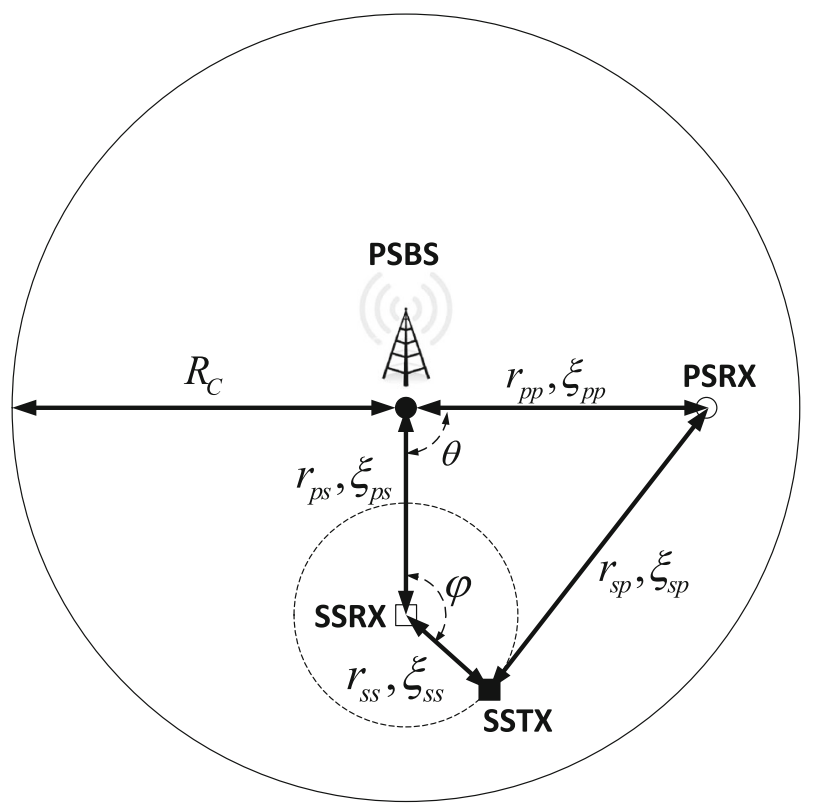

Fig. 1 Geographical parameters among the PS and SS nodes in a single-cell network system 
while the second corresponds to the receiver. A primary transmitter (base station PSBS) is located at the center of the primary network with radius $R_{C}$. It is assumed that primary system receiver (PSRX) and secondary system receiver (SSRX) are independently uniformly distributed inside the primary service area. Furthermore, secondary system transmitter (SSTX) is assumed to reside uniformly anywhere on a circle centered at the SSRX with radius $r_{s s}$. Under this probabilistic setting, the joint probability density function (PDF) of the inter-node distances and angles can be expressed as

$$
\operatorname{Pr}\left(r_{p s}, r_{p p}, \theta, \phi\right)=\frac{2 r_{p s} r_{p p}}{\pi^{2} R_{C}^{4}},
$$

for $0 \leq r_{p s}, r_{p p} \leq R_{C}, 0 \leq \theta \leq \pi$, and $0 \leq \phi \leq 2 \pi$, where $\theta$ is the angle between $r_{p p}$ and $r_{p s}$, and $\phi$ is the angle between $r_{s s}$ and $r_{p s}$. Both angles are uniformly distributed in their respective domains. From the network topology depicted in Fig. 1, $r_{s p}$ can be determined for each realization of these random variables as $r_{s p}=\left|r_{p p} e^{i \theta}-\left(r_{p s}+r_{s s} e^{i(\pi-\phi)}\right)\right|$, where $r_{s s}$ is assigned different values for capacity computations.

We denote the state vector for node locations with $\mathbf{n}=\left[r_{p s}, r_{p p}, \theta, \phi\right]$. Using the same notation, the state vector for shadow fading is represented by $\xi=\left[\xi_{s s}, \xi_{s p}, \xi_{p s}, \xi_{p p}\right]$. Elements of $\boldsymbol{\xi}$ are modeled as independent and identically distributed (i.i.d.) Gaussian random variables with zero mean and standard deviation $\sigma_{d B}$, where subscript $d B$ relates to the well-known log-normal shadowing model [19]. $\boldsymbol{\xi}$ and $\mathbf{n}$ are assumed to be statistically independent, meaning that shadow fading is independent of the distribution of the nodes within the primary network. Hence, the combined effect of path loss and shadow fading is modeled as follows

$$
G\left(r, \xi_{T X, R X}\right)=G_{T X} G_{p a t h, T X, R X}(r) 10^{\frac{\xi_{T X, R X}}{10}} G_{R X}
$$

where $G_{\text {path } T X, R X}(r)$ is the contribution of path gain $(\leq 1)$ alone, $10^{\xi_{T X, R X} / 10}$ is the gain due to log-normal shadowing, $G_{T X}$ and $G_{R X}$ are antenna gains at the transmitter and receiver, respectively. When one of the nodes in the transmitter-receiver link is the base station, the path gain model is denoted by $G_{\text {path, } B, U E}(r)$, which is computed based on ITU-R M. 1225 Pedestrian [20]. For the remaining links, a common path gain model based on IEEE 802.11n Model $F$ is assumed and represented by $G_{p a t h, U E, U E}(r)$, where UE means user equipment [21].

The primary transmit power $P_{p}$ is fixed, as is usually the case for base stations [19]. The allocation policy for the secondary transmit power $P_{s}$ will be determined in this paper relying only on the instantaneous value of the shadow fading between secondary transmitter and primary receiver in the absence of any path loss estimates. ${ }^{1}$ Although only partial CSI is required at the SS, the information regarding the instantaneous shadow fading should still be communicated between PS and SS through reliable links (e.g., a wired backbone channel, a wireless channel such as a cognitive pilot channel, or a common database server). For clarity of discussion, thermal noise at the SS receiver is assumed to be negligible in comparison with the interference from PS user. Finally, this discussion can be generalized easily to multiple primary receivers by restricting primary transmitter to communicate with a single primary receiver at any given time; hence, the interference within the PS due to primary agents is avoided.

\footnotetext{
1 Section 4 discusses the approaches on how to estimate the instantaneous value of the shadow fading.
} 


\section{Secondary System Transmission Power Control under Average SINR Loss Constraint}

When the SS utilizes the downlink (DL) resources of the PS, the victim of the interference from the SS is a PS user equipment (PSRX) as shown in Fig. 1. To control the interference level for the target PS, a method of transmission power control is needed for the SSTX. If the PS can tolerate an average SINR loss $\mathrm{SINR}_{\text {loss,tol }}$, caused by the interference from the SS, the value SINR $_{\text {loss, tol }}$ can be regarded as the SINR margin of the PS. For example, the SINR margin of the PS could be obtained from the difference between the actual SINR of the target primary link and the required SINR in accordance with the Quality of Service (QoS) level of the target primary link. Alternatively, an interference level for the target primary link that is considered to be negligible could be regarded as the SINR margin of the PS. Hence, the value $\mathrm{SINR}_{\text {loss, tol }}$, which corresponds to the SINR margin of the PS, is an important parameter in this method.

The ergodic capacity maximization problem for the SS under an average SINR loss constraint can be written as

$$
\begin{aligned}
\max _{P_{s}(\mathbf{n}, \boldsymbol{\xi})} & \mathbb{E}_{\mathbf{n}, \boldsymbol{\xi}}\left\{\log _{2}\left(1+\mathrm{SIR}_{s}\right)\right\} \\
\text { subject to } & \mathbb{E}_{\mathbf{n}, \boldsymbol{\xi}}\left\{\operatorname{SINR}_{\mathrm{p}, \text { loss }}\right\} \leq \mathrm{SINR}_{\text {loss }, \text { tol }},
\end{aligned}
$$

where $P_{S}(\mathbf{n}, \boldsymbol{\xi})$ denotes the power transmission strategy of the SS assuming full CSI is available, $\operatorname{SIR}_{s}$ represents secondary signal to primary interference ratio at the secondary receiver, $\mathrm{SINR}_{\mathrm{p}, \text { loss }}$ denotes primary signal to secondary interference plus noise ratio at the primary receiver, and $\mathrm{SINR}_{\text {loss, tol }}$ represents the average SINR loss tolerance at the primary receiver.

The instantaneous SINR loss at the primary receiver is given as

$$
\mathrm{SINR}_{\mathrm{p}, \text { loss }}=1+\frac{P_{s}(\mathbf{n}, \boldsymbol{\xi}) G\left(r_{s p}, \xi_{s p}\right)}{N_{p}},
$$

where $N_{p}$ denotes the thermal noise power at the PS receiver. The detailed derivation of (4) is shown in the section "Derivation of Instantaneous SINR Loss at PS receiver" of "Appendix". It is noted that $\mathrm{SINR}_{\mathrm{p}, \text { loss }}$ is independent of the primary signal power $P_{p}$. Similarly, we have

$$
\mathrm{SIR}_{s}=\frac{P_{s}(\mathbf{n}, \boldsymbol{\xi}) G\left(r_{s s}, \xi_{s s}\right)}{P_{p} G\left(r_{p s}, \xi_{p s}\right)},
$$

Using the method of Lagrange multipliers [19, Chapter 4], the optimal transmission power allocation policy for SS is given as

$$
P_{s}(\mathbf{n}, \boldsymbol{\xi})=\left[c \frac{N_{p}}{G\left(r_{s p}, \xi_{s p}\right)}-\frac{P_{p} G\left(r_{p s}, \xi_{p s}\right)}{G\left(r_{s s}, \xi_{s s}\right)}\right]^{+},
$$

where $[x]^{+}=\max (0, x)$ and $c$ is a constant that should be determined from the average SINR loss constraint in (3). Since the expectation needs to be taken over both $\mathbf{n}$ and $\xi$, it is very difficult to compute in general. Even if it is solved, optimal strategy requires perfect CSI at the secondary transmitter which is not practically desirable.

In the following, we assume that the only CSI available to SS is the instantaneous value of the shadow fading between secondary transmitter and primary receiver. Based on this assumption, we show that an upper bound on the capacity can be obtained by invoking Jensen's inequality [22]. Let $P_{s}\left(r_{s s}, \xi_{s p}\right)$ denote secondary transmission power as a function 
of $\xi_{s p}$ when secondary transmitter and receiver are separated by a distance $r_{s s}$. Since $\mathbf{r}$ and $\xi$ are independent, average SINR loss at the primary receiver can be expressed as

$$
\overline{\mathrm{SINR}_{\mathrm{p}, \operatorname{loss}}}=1+\kappa\left(r_{s s}\right) \int_{-\infty}^{\infty} P_{s}\left(r_{s s}, \xi\right) 10^{\frac{\xi}{10}} f_{\xi_{s p}}(\xi) d \xi
$$

where $\kappa\left(r_{s s}\right) \triangleq G_{U E}^{2} / N_{p} \mathbb{E}_{\mathbf{n}}\left\{G_{\text {path }, U E, U E}\left(r_{s p}\right)\right\}, f_{\xi_{s p}}(\xi)$ is the zero mean Gaussian distribution with standard deviation $\sigma_{d B}$ for shadow fading exponent, and the expectation is computed over the joint PDF of the inter-node distances as stated in (1). Subsequently, average SIR at the secondary receiver can be obtained as a function of the shadow fading exponent $\xi_{s p}$ and the distance $r_{s s}$ as $\overline{\operatorname{SIR}_{s}\left(r_{s s}, \xi_{s p}\right)}=P_{s}\left(r_{s s}, \xi_{s p}\right) \chi\left(r_{s s}\right)$, where

$$
\chi\left(r_{s s}\right) \triangleq \frac{G_{U E} e^{\left(\frac{\ln 10}{10} \sigma_{d B}\right)^{2}}}{P_{p} G_{B S}} G_{p a t h, U E, U E}\left(r_{s S}\right) \mathbb{E}_{\mathbf{n}}\left\{\frac{1}{G_{p a t h, B S, U E}\left(r_{p s}\right)}\right\} .
$$

From Jensen's inequality, an upper bound on the ergodic capacity can now be obtained as

$$
\mathrm{C}_{\mathrm{s}}^{\mathrm{up}}\left(r_{s s}\right)=\int_{-\infty}^{\infty} \log _{2}\left(1+\overline{\operatorname{SIR}_{s}\left(r_{s s}, \xi_{s p}\right)}\right) f_{\xi_{s p}}(\xi) d \xi \mathrm{bps} / \mathrm{Hz}
$$

In the following discussion, the subscripts are dropped to preserve notational simplicity. In order to obtain transmit power strategy maximizing the upper bound on the capacity for SS, the following constrained optimization problem is constructed

$$
\begin{aligned}
\max _{P_{s}(\xi)} & \int_{-\infty}^{\infty} \log _{2}\left(1+P_{S}(\xi) \cdot \chi\right) f(\xi) d \xi \\
\text { subject to } & \int_{-\infty}^{\infty} P_{S}(\xi) 10^{\frac{\xi}{10}} f(\xi) d \xi \leq \gamma,
\end{aligned}
$$

where $\gamma \triangleq\left(\operatorname{SINR}_{\text {loss }, \text { tol }}-1\right) / \kappa$ is obtained from the constraint on the average SINR loss. We can write the Lagrangian as

$$
L\left(P_{S}(\xi), \lambda\right)=-\int_{-\infty}^{\infty} \log _{2}\left(1+P_{S}(\xi) \cdot \chi\right) f(\xi) d \xi+\lambda\left(\int_{-\infty}^{\infty} P_{S}(\xi) 10^{\frac{\xi}{10}} f(\xi) d \xi-\gamma\right)
$$

and differentiating with respect to $P_{S}(\xi)$, we get

$$
\begin{aligned}
\frac{\partial L\left(P_{S}(\xi), \lambda\right)}{\partial P_{S}(\xi)} & =-\frac{1}{\ln 2} \frac{\chi}{1+P_{S}(\xi) \chi} f(\xi)+\lambda 10^{\frac{\xi}{10}} f(\xi)=0 \\
\Longrightarrow P_{S}(\xi) & =\frac{1}{10^{\frac{\xi}{10}} \lambda \ln 2}-\frac{1}{\chi} .
\end{aligned}
$$

Solving for $P_{S}(\xi)$ with the constraint that $P_{S}(\xi) \geq 0$ yields the familiar water-filling solution as

$$
P_{s}\left(r_{s s}, \xi_{s p}\right)= \begin{cases}\frac{1}{\chi}\left(10^{\frac{\xi_{0}-\xi_{s p}}{10}}-1\right) & \text { if } \xi_{s p}<\xi_{0} \\ 0 & \text { if } \xi_{s p} \geq \xi_{0}\end{cases}
$$


for some cut-off value $\xi_{0}$. It is noted that SS transmission is stopped whenever the shadow fading exponent $\xi_{s p}$ between secondary transmitter and primary receiver exceeds the cut-off value $\xi_{0}$ (in $\mathrm{dB}$ ) in order to satisfy the average SINR loss constraint at the primary receiver. $\xi_{0}$ can be solved numerically from the interference constraint, i.e.,

$$
\int_{-\infty}^{\xi_{0}}\left(10^{\frac{\xi_{0}}{10}}-10^{\frac{\xi}{10}}\right) f(\xi) d \xi=\chi \cdot \gamma
$$

Since normal distribution with zero mean and standard deviation $\sigma_{d B}$ is assumed for the shadow gain $\xi_{s p}$ between SSTX and PSRX, the cut-off value can be solved equivalently from

$$
10^{\xi_{0} / 10}\left(1-\mathcal{Q}\left(\frac{\xi_{0}}{\sigma_{d B}}\right)\right)-e^{\left(\frac{\ln 10}{10} \sigma_{d B}\right)^{2} / 2}\left(1-\mathcal{Q}\left(\frac{\xi_{0}}{\sigma_{d B}}-\frac{\ln 10}{10} \sigma_{d B}\right)\right)=\chi \cdot \gamma
$$

where $\mathcal{Q}(\cdot)$ represents the $\mathcal{Q}$-function for the tail probability of the standard normal distribution. It should be emphasized that since $\chi$ is a function of $r_{s s}, \xi_{0}$ also depends on $r_{s s}$ by the above equation.

The power adaptation strategy given in (13) allows SSTX to adjusts its transmit power based only on the instantaneous value of the shadow fading exponent between SSTX and PSRX. In other words, the power adaptation can be performed in the absence of any path loss estimates which would require additional knowledge of the distance between the respective nodes. Section 4 discusses the approaches on how to estimate the instantaneous value of the shadow fading.

\section{Estimation of Shadow Fading}

In this part, we present a method for estimating the instantaneous value of the shadow fading exponent which does not require the knowledge of the distance between secondary transmitter and primary receiver (hence, it is applicable in the absence of any path loss estimates). Our approach is based on the modification of the least-squares shadow fading estimation method that is discussed in the references $[23,24]$. We begin by summarizing the method proposed in $[23,24]$.

\subsection{Least Squares Based Shadow Fading Estimation}

The first step in the estimation of the shadow fading is to eliminate the multipath effect in the received signal power. Since the fast fading due to multipath scattering varies with a distance on the order of a wavelength, averaging the received power over segments of $30 \lambda$ can remove small-scale effects such as multipath fading while large-scale effects such as distance loss and shadow fading can be assumed to stay constant [25].

Next, a deterministic distance dependent path loss model similar to Okumura-Hata model [26] is assumed in order to extract the shadow fading component:

$$
10 \log _{10}\left(h_{\text {loss }}(d)\right)=A+B \log _{10}(d)
$$

where $d$ is the distance between secondary transmitter and primary receiver (in $\mathrm{km}$ ), $h_{\text {loss }}$ is the deterministic long term distance dependent path loss. Together with contribution from the shadow fading, the integral (overall) path loss is expressed as

$$
10 \log _{10}\left(h_{c h}(d)\right)=A+B \log _{10}(d)+10 \log _{10}\left(h_{s h}\right)
$$


where $h_{s h}$ is the channel's shadow fading component (modeled with log-normally distributed random variable) that is responsible for the slow variation in the received signal power due to obstacles and obstruction in the propagation path. Recalling that the random variable describing the shadow fading component is modelled as

$$
h_{s h}=10^{Z \sigma_{s h} / 10}
$$

where $Z$ is a zero-mean Gaussian random variable with unit variance, we have

$$
\mathrm{E}\left\{10 \log _{10}\left(h_{s h}\right)\right\}=0 d B
$$

Measurement of the integral path loss is obtained over each segment and the data set $\left\{10 \log _{10}\left(h_{c h}\left(d_{i}\right)\right) ; d_{i}\right\}$ is constructed. When a large number of measurements are collected, the distance loss component can be estimated by calculating the least squares fit to the average received powers from all $30 \lambda$ segments against log-distance. In other words, the parameters $A$ and $B$ can be obtained as the least squares estimate (c.f. least squares line fitting problem [27]). Using the regression equation (i.e., parameters $A$ and $B$ ), the shadow fading component can be extracted as the vertical distance between the estimated distance dependent path loss component (i.e., the regression line) and the average received power measurement over each local area $[23,24]$.

\subsection{Proposed Shadow Fading Estimation Method}

In this part, we adapt the least squares based shadow fading estimation method described in the previous paragraphs so that it does not require the knowledge of the exact distance between transmitter and receiver. Instead, we assume that the average received powers from all $30 \lambda$ segments are measured with respect to an unknown baseline distance $d_{0}$ between transmitter and receiver. ${ }^{2}$ Using the same path loss model as above, the following relationship is obtained:

$$
\begin{aligned}
10 \log _{10}\left(h_{c h}(d)\right) & =A+B \log _{10}\left(d_{0}+d_{i}\right)+10 \log _{10}\left(h_{s h}\right) \\
& =A+B \log _{10}\left(d_{0}\right)+B \log _{10}\left(1+\frac{d_{i}}{d_{0}}\right)+10 \log _{10}\left(h_{s h}\right)
\end{aligned}
$$

By the following Taylor expansion identity, $\ln (1+x)=x-\frac{x^{2}}{2}+\frac{x^{3}}{3}-\frac{x^{4}}{4}+\cdots$ for $-1<x \leq 1$ and keeping the first two terms in the above expression, we obtain the second order Taylor approximation:

$$
\log _{10}\left(1+\frac{d_{i}}{d_{0}}\right) \approx \frac{1}{\ln 10}\left(\frac{d_{i}}{d_{0}}-\frac{d_{i}^{2}}{2 d_{0}^{2}}\right)
$$

By defining $a_{0} \triangleq A+B \log _{10}\left(d_{0}\right), a_{1} \triangleq \frac{B}{d_{0} \ln 10}$ and $a_{2} \triangleq \frac{-B}{2 d_{0}^{2} \ln 10}$, the integral path loss can be expressed as

$$
10 \log _{10}\left(h_{c h}(d)\right) \approx a_{0}+a_{1} d_{i}+a_{2} d_{i}^{2}+10 \log _{10}\left(h_{s h}\right)
$$

or similarly by keeping the first $n$ terms, the integral path loss can be approximated by the following $n$th order polynomial:

$$
10 \log _{10}\left(h_{c h}(d)\right) \approx a_{0}+a_{1} d_{i}+a_{2} d_{i}^{2}+\cdots+a_{n} d_{i}^{n}+10 \log _{10}\left(h_{s h}\right)
$$

2 Contrary to the previous case, in this framework it is necessary that the secondary transmitter moves along the line connecting the secondary transmitter to primary receiver in order to express the total distance as $d_{0}+d_{i}$ 
Consequently, with a large number of measurements, we can obtain a new least squares fit to the average powers over all $30 \lambda$ segments (corresponding to various $d_{i}$ values such that $\left|d_{i}\right|<d_{0}$ ). Contrary to the previous case, this estimate depends on the distances between the initial measurement location of the secondary transmitter and the consecutive segments over which measurements are conducted instead of the exact distance between secondary transmitter and primary receiver at each successive measurement segment.

In matrix notation,

$$
\underbrace{\left[\begin{array}{l}
P_{1} \\
P_{2} \\
\vdots \\
P_{m}
\end{array}\right]}_{\mathbf{p}}=\underbrace{\left[\begin{array}{lllll}
1 & d_{1} & d_{1}^{2} & \cdots & d_{1}^{n} \\
1 & d_{2} & d_{2}^{2} & \cdots & d_{2}^{n} \\
\vdots & & & \vdots & \vdots \\
1 & d_{m} & d_{m}^{2} & \cdots & d_{m}^{n}
\end{array}\right]}_{\mathbf{D}} \underbrace{\left[\begin{array}{c}
a_{0} \\
a_{1} \\
\vdots \\
a_{n}
\end{array}\right]}_{\mathbf{a}}
$$

where $\mathbf{p}$ represents the average received power measurements, $\mathbf{D}$ is the matrix of relative distances of the measurement locations with respect to the initial reference point, and $\mathbf{a}$ is the regression polynomial coefficients. When the number of measurements $m$ is much larger than the number of regression coefficients $n+1$, the least squares solution is given by

$$
\hat{\mathbf{a}}=\left(\mathbf{D}^{T} \mathbf{D}\right)^{-1} \mathbf{D}^{T} \mathbf{p}
$$

As discussed previously, once the regression coefficients $\left\{a_{0}, a_{1}, a_{2}, \ldots, a_{n}\right\}$ are computed, the shadow fading component $10 \log _{10}\left(h_{\text {shadow }}\right)$ can be determined from the vertical distance between the measured average received power and the distance dependent path loss estimate calculated from the regression polynomial for a given relative distance $d_{i}$. Implicit in the derivations, it is assumed that the transmitter power is fixed while the received power measurement are taken.

It should be pointed out that the above approach takes into account the effect of distance dependent path loss over the segments where average (over the multipath fading) received power measurements are collected. This analysis can be simplified even further if we can assume that the distance dependent path loss can be safely assumed constant across these segments while assuring that they are well-separated to obtain uncorrelated shadow fading measurements. In this case, the regression operation which basically provides us with the distance dependent path loss information is no longer necessary. In other words, after the local power measurements are obtained by averaging over the multipath effect in each segment, the distance dependent path loss value can be computed by carrying out a final averaging operation over the values returned from each segment. Lastly, the instantaneous shadow fading component can be determined by subtracting the received power measurement from the computed mean value. Using the same notation as above, the mean received power due to distance dependent path loss effect (averaged over multipath and shadow fading) is calculated by the sample average over the $\mathrm{dB}$ values as follows

$$
\bar{P}=\frac{\sum_{k=1}^{m} P_{k}}{m}
$$

Next, let $P_{\text {inst }}$ denote the instantaneous local received power averaged over the multipath fading. The instantaneous shadow fading loss is given by

$$
10 \log _{10}\left(h_{\text {shadow }}\right)=P_{\text {inst }}-\bar{P}
$$


Table 1 System model parameters

\begin{tabular}{ll}
\hline Parameter & Value \\
\hline PS service area $\left(R_{C}\right)$ & $500 \mathrm{~m}$ \\
Operating frequency $(f)$ & $2,500 \mathrm{MHz}$ \\
Average SINR loss $\left(\mathrm{SINR}_{\text {loss }, t o l}\right)$ & $0.01 \mathrm{~dB}$ \\
Transmission power of PSBS $\left(P_{p}\right)$ & $20,30,40 \mathrm{dBm}$ \\
PSRX noise power $\left(N_{p}\right)$ & $-96.8 \mathrm{dBm}$ \\
BS/UE antenna gain $\left(G_{B S} / G_{U E}\right)$ & $10 / 0 \mathrm{~dB}$ \\
Shadow fading SD $\left(\sigma_{d B}\right)$ & $10 \mathrm{~dB}$ \\
\hline
\end{tabular}

\subsection{Related Resources}

In the previous paragraphs, we have focused on the estimation of the instantaneous shadow fading component from the received power measurements using the least squares approach. The first step in this method was to average the instantaneous received power to remove the fast multipath fading while following the variations of the slower shadow fading. Based on the work in [23,24], the averaging window size was selected to be $30 \lambda$. However, depending on the relative velocity between transmitter and receiver, shadow fading correlation and other considerations, the averaging filter bandwidth may need to be updated [28, Sec. 12.3]. For a more detailed discussion on window-based estimators, we refer the reader to the review paper [29]. Window-based estimators are designed assuming constant shadow power over the duration of an averaging window [30-32]. There are also Kalman filter based power estimation and prediction algorithms with superior performance in comparison to windowbased approaches [33]. The non-Gaussian nature of the received log-powers requires special consideration in wireless radio environments. To that aim, a sequential Bayesian method is proposed in [34] for dynamic estimation and prediction of local mean powers from instantaneous signal powers in composite fading-shadowing channels with a Nakagami-m fading component and $\mathrm{AR}(1)$ shadowing component.

\section{Numerical Results}

In this section, we present several numerical simulations in order to evaluate the performance of the proposed power adaptation strategy under the average SINR loss constraint. System model parameters are selected as shown in Table 1.

The distance gain $G_{\text {path }, B S, U E}(r)$ between BS and UE is modeled based on ITU-R M. 1225 [20]. Since path gain should always be less than the free space gain, we have slightly modified this model to prevent the formula resulting in high gain factors for small values of the distance $r$. The resulting path gain formula can be expressed as a piecewise function

$$
G_{\text {path }, B S, U E}(r)= \begin{cases}\frac{10^{7.1}}{r^{4} f^{3}} & \text { if } r \geq r_{1} \\ \frac{10^{7.1}}{r_{1}^{4} f^{3}} & \text { if } r<r_{1}\end{cases}
$$

where $r$ is in meters and $f$ is in MHz. $r_{1}$ can be chosen as a small fraction of the PS cell radius. In our analysis, $r_{1}=0.01 R_{C}$ is employed.

The distance gain $G_{p a t h, U E, U E}(r)$ between UEs is modeled according to IEEE 802.11n model $\mathrm{F}$ [21], which is described by the following piecewise function (after the slight modification explained above) 


$$
G_{\text {path }, U E, U E}(r)= \begin{cases}\left(\frac{c}{4 \pi 10^{6} f}\right)^{2} \cdot \frac{30^{1.5}}{r^{3.5}} & \text { if } r \geq 30 \\ \left(\frac{c}{4 \pi 10^{6} f r}\right)^{2} & \text { if } r_{1} \leq r<30 \\ \left(\frac{c}{4 \pi 10^{6} f r_{1}}\right)^{2} & \text { if } r<r_{1}\end{cases}
$$

where $r, f, r_{1}$ are as defined above, and $c$ is the speed of light in meters/sec.

The next constituent of the path gain, i.e., the shadow gain $\xi_{i j}$ between any two nodes $i$ and $j$ is modeled as a Gaussian random variable with zero mean and standard deviation $\sigma_{d B}=10 \mathrm{~dB}$. No correlation is assumed among shadow gains corresponding to distinct node pairs, hence implying independent yet identical Gaussian models with the following PDF

$$
p_{\xi_{i j}}(\xi)=\frac{1}{\sqrt{2 \pi} \sigma_{d B}} e^{-\xi^{2} / 2 \sigma_{d B}^{2}},
$$

for $-\infty<\xi<\infty$.

The exact ergodic capacity as a function of $r_{s s}$ can then be computed numerically from

$$
\begin{aligned}
\mathrm{C}_{\mathrm{s}}^{\text {exact }}\left(r_{s s}\right) & =\mathbb{E}_{\mathbf{n}, \xi}\left\{\log _{2}\left(1+\operatorname{SIR}_{s}\right)\right\} \\
& =\mathbb{E}_{\mathbf{n}, \xi}\left\{\log _{2}\left(1+\frac{P_{s}\left(r_{s s}, \xi_{s p}\right) G\left(r_{s s}, \xi_{s s}\right)}{P_{p} G\left(r_{p s}, \xi_{p s}\right)}\right)\right\}
\end{aligned}
$$

where $P_{s}\left(r_{s s}, \xi_{s p}\right)$ is substituted from (13). The multiple integral over the joint pdf of the inter-node distances, angles and shadow fading distributions are evaluated numerically by averaging the results from a total of $10^{7}$ realizations of each random quantity using Monte Carlo integration techniques [35].

\subsection{Exact Ergodic Capacity Analysis}

In Table 2, the parameters necessary for calculating the proposed power adaptation strategy while satisfying the average SINR loss constraint are provided for downlink communications. For a given value of $r_{s s}$, transmit power of SS is determined by substituting the parameters supplied in the corresponding row of the table into (13).

Next, we provide the plots for the exact ergodic capacity curves which are obtained by computing the instantaneous SIR from (31) based on the power adaptation parameters given in Table 2. Using this quantity, we obtain the corresponding instantaneous capacity values which are then averaged over the joint PDF of the inter-node distances and the shadow fading distribution as suggested by (31).

The resulting exact Shannon capacity curves are depicted in Fig. 2. Relatively small capacity values can be attributed to the fact that the power adaptation strategy utilizes only the knowledge of instantaneous shadow fading whereas full CSI of the links in the single-cell environment is required to attain higher capacity values.

Further insight can be obtained by inspecting Fig. 2 in more detail. As the transmit power of PSBS increases, SS ergodic capacity decreases due to higher levels of interference from PS. More evidently, SS ergodic capacity decreases with increasing distance between SSTX and SSRX due to higher path loss.

\subsection{Effect of Shadow Fading Exponent Standard Deviation}

In this part, we try to find out how the performance of the proposed power adaptation strategy responds to changes in the standard deviation of the shadow fading exponent. To that 
Table 2 Power adaptation parameters for DL communications scenario in Fig. 2

\begin{tabular}{|c|c|c|c|c|c|c|}
\hline \multirow[t]{2}{*}{$r_{s s}(\mathrm{~m})$} & \multicolumn{2}{|c|}{$P_{p}=20 \mathrm{dBm}$} & \multicolumn{2}{|c|}{$P_{p}=30 \mathrm{dBm}$} & \multicolumn{2}{|c|}{$P_{p}=40 \mathrm{dBm}$} \\
\hline & $\chi$ & $\xi_{0}$ & $\chi$ & $\xi_{0}$ & $\chi$ & $\xi_{0}$ \\
\hline 10 & $4,737,420$ & 30.49 & 473,742 & 20.74 & 47,374 & 11.66 \\
\hline 20 & $1,192,817$ & 24.60 & 119,282 & 15.19 & 11,928 & 6.71 \\
\hline 30 & 536,380 & 21.26 & 53,638 & 12.13 & 5,364 & 4.03 \\
\hline 40 & 199,296 & 17.22 & 19,930 & 8.51 & 1,993 & 0.88 \\
\hline 50 & 93,130 & 14.23 & 9,313 & 5.87 & 931 & -1.42 \\
\hline 60 & 50,453 & 11.90 & 5,045 & 3.83 & 505 & -3.19 \\
\hline 70 & 30,259 & 10.01 & 3,026 & 2.18 & 303 & -4.63 \\
\hline 80 & 19,595 & 8.45 & 1,960 & 0.83 & 196 & -5.81 \\
\hline 90 & 13,453 & 7.13 & 1,345 & -0.32 & 135 & -6.82 \\
\hline 100 & 9,662 & 5.99 & 966 & -1.31 & 97 & -7.69 \\
\hline 110 & 7,219 & 5.01 & 722 & -2.16 & 72 & -8.44 \\
\hline 120 & 5,563 & 4.15 & 556 & -2.91 & 56 & -9.10 \\
\hline 130 & 4,403 & 3.38 & 440 & -3.58 & 44 & -9.69 \\
\hline 140 & 3,561 & 2.70 & 356 & -4.17 & 36 & -10.22 \\
\hline 150 & 2,940 & 2.09 & 294 & -4.70 & 29 & -10.69 \\
\hline 160 & 2,468 & 1.54 & 247 & -5.19 & 25 & -11.12 \\
\hline 170 & 2,102 & 1.04 & 210 & -5.62 & 21 & -11.51 \\
\hline 180 & 1,814 & 0.59 & 181 & -6.02 & 18 & -11.86 \\
\hline 190 & 1,585 & 0.18 & 159 & -6.38 & 16 & -12.18 \\
\hline 200 & 1,398 & -0.20 & 140 & -6.72 & 14 & -12.48 \\
\hline
\end{tabular}

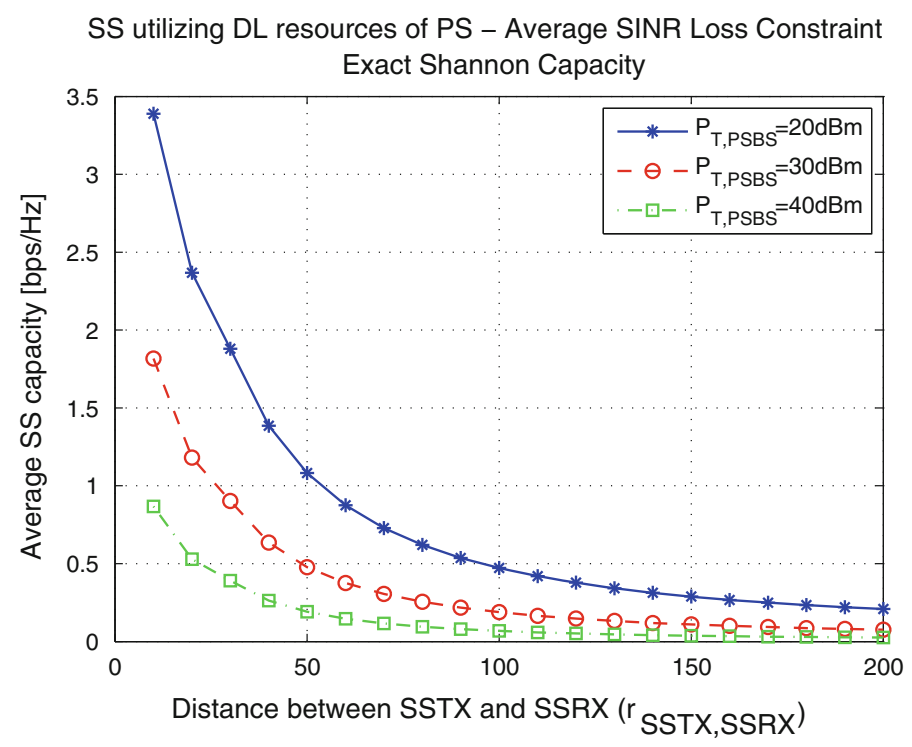

Fig. 2 Exact ergodic capacity versus distance between SSTX and SSRX under average SINR loss constraint using the power adaptation strategy depicted in Table 2 for downlink communications, (SINR loss, tol $=0.01 \mathrm{~dB}$ ) 
SS utilizing DL resources of PS - Power Adaptation Strategy

Effect of Shadow Fading Exponent Standard Deviation

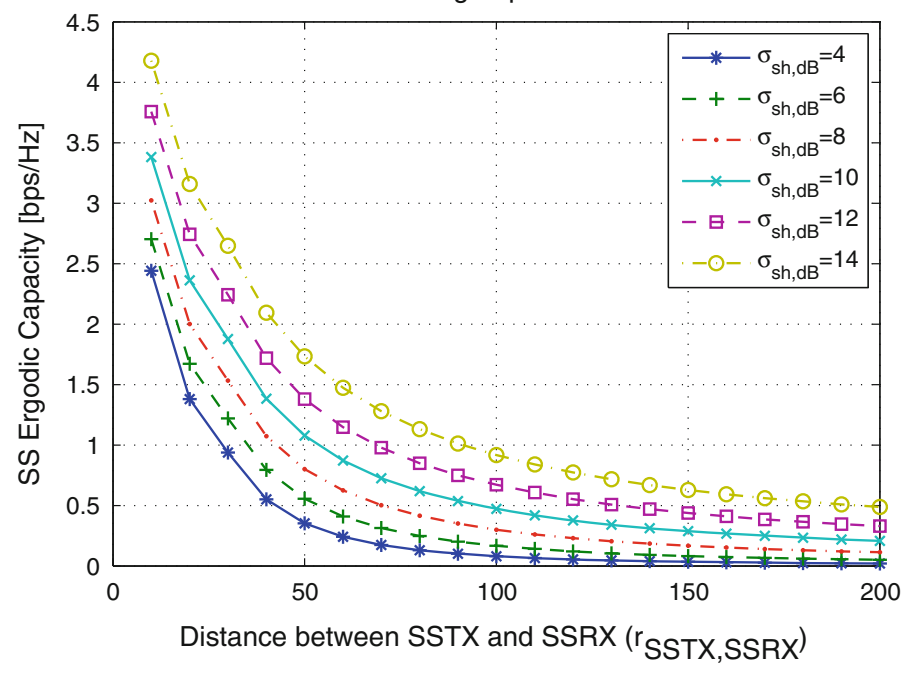

Fig. 3 Exact ergodic capacity versus distance between SSTX and SSRX for various values of the shadow fading exponent standard deviation using the power adaptation strategy for downlink communications under the same average SINR loss constraint $\left(\mathrm{SINR}_{\text {loss }, \text { tol }}=0.01 \mathrm{~dB}\right)$

aim, we let the standard deviation of the shadow fading exponent to take values in the set $\{4,6,8,10,12,14\} \mathrm{dB}$. Assuming i.i.d. shadow fading between the nodes, the proposed power adaptation strategy is implemented assuming this information is available at the SS. Same procedure is repeated for all values of the standard deviation of the shadow fading exponent. The resulting average ergodic capacity values are plotted versus the distance between SSTX and SSRX in Fig. 3. It is observed that the performance of the power adaptation strategy improves as the standard deviation of the shadow fading exponent is increased. This fact can be partly anticipated by noting that as the variance of the shadow fading increases, some of the instantaneous shadow fading measurements that are smaller than the threshold value $\xi_{0}$ may have much lower values which will in turn have significant contribution to the ergodic capacity due to the exponential nature of the power assignment function given in (13). As a conclusion, we can state that the water filling power adaptation strategy favors the shadowing processes with higher variances over shadowing processes with lower variances under the same average SINR loss constraint on the condition that the instantaneous shadow fading measurements are error-free.

\subsection{Effect of Shadow Fading Exponent Estimation Error}

In this part, we analyze the effect of erroneously estimating the instantaneous value of the shadow fading exponent on the performance of our power adaptation strategy. We try to find out the effects on the ergodic capacity of the secondary system as well as the effects on the SINR loss induced at the primary system receiver. To that aim, the error incurred in estimating the instantaneous value of the shadow fading exponent between SSTX and PSRX is modeled as a white uniform random variable added independently to true value of the instantaneous shadow fading exponent. The proposed power adaptation strategy is obtained based on the true value of the shadow fading standard deviation, but the resulting 
hard-coded power assignment function is supplied with the noise corrupted instantaneous shadow fading exponent measurements. The resulting ergodic capacity of the SS and the SINR loss at the PS are calculated by integrating the respective formulae over the joint PDF of the inter-node distances and the true shadow fading distribution. Since both the shadow fading exponent and the estimation error are modeled as independently distributed random variables, the measured shadow fading exponent (which is defined as the sum of the two aforementioned quantities) is also a random variable with variance equal to the sum of the component variances.

The standard deviation of the shadow fading exponent estimation error is controlled through a multiplicative constant $q$. More explicitly, the variance of the measured shadow fading exponent is given as

$$
\sigma_{\text {sh,measured }}^{2}=q \cdot \sigma_{\text {err }}^{2}+\sigma_{\text {sh,true }}^{2}
$$

The variance of the uniformly generated estimation errors is chosen to be equal to the true variance of the shadow fading exponent, i.e., $\sigma_{e r r}^{2}=\sigma_{s h, t r u e}^{2}$. By assigning different values to the parameter $q$, the intensity of the shadow fading estimation error is adjusted. Evidently, $q=0$ results in error-free instantaneous shadow fading measurements (or estimates), and it is enough to consider only the positive values for $q$ since the estimation errors are generated as zero-mean uniform random variables.

In Fig. 4a, we plot the effect of the shadow fading exponent estimation error on the exact ergodic capacity of the link between SSTX and SSRX for downlink communications with $P_{p}=20 \mathrm{dBm}$. It is observed that the capacity drops gracefully for a given value of the distance between SSTX and SSRX as the variance of the estimation error is increased. This is somewhat expected because estimation errors are uniformly distributed zero mean random variables whereas true shadow fading exponent values are zero mean Gaussian random variables. With a positive value for the threshold $\xi_{0}$, as the variance of estimation error increases, more shadow fading samples will exceed the threshold $\xi_{0}$ when added to the estimation errors (causing the transmission to be aborted, i.e. $P_{s}=0$ ) than the ones shifted below the threshold.

In Fig. 4b, the average SINR loss of the primary system due to SS transmission is depicted as the variance of the estimation error is changed for various distance values between SSTX and SSRX. Although the ergodic capacity is decreasing with increasing estimation error, it is noted that the average SINR loss induced at the PSRX changes very little (close to the target value $\mathrm{SINR}_{\mathrm{p} \text {, loss }}=0.01 \mathrm{~dB}$ ) as we increase the power of the noise up to twice the variance of the shadow fading exponent. If the noise power is increased to even higher values with respect to the true value of the shadow fading exponent's variance, we expect that the average SINR loss would rise to intolerable values jeopardizing the robust behavior of the power assignment strategy. This is mainly due to the fact that in this case, measurement noise would dominate the shadow fading measurements and negative values of high magnitude would result in exponentially increasing power assignments and cause significant interference to PS receiver as can be deduced from the power adaptation strategy given in (13). However, we have plotted up to twice the shadow fading variance ( $\sim 1.41$ of the shadow fading standard deviation) in $\mathrm{dB}$. This choice is due to the fact that higher measurement noise power values do not conform with practical cases. Also, note that the z-axis corresponding to average SINR loss is not in $\mathrm{dB}$ units.

In order to thoroughly understand the probabilistic structure of the SINR loss at the PSRX due to SS transmission, the empirical cumulative distribution function and some important statistics of the SINR loss are presented for $r_{s s}=50 \mathrm{~m}$ and measurement noise coefficient 


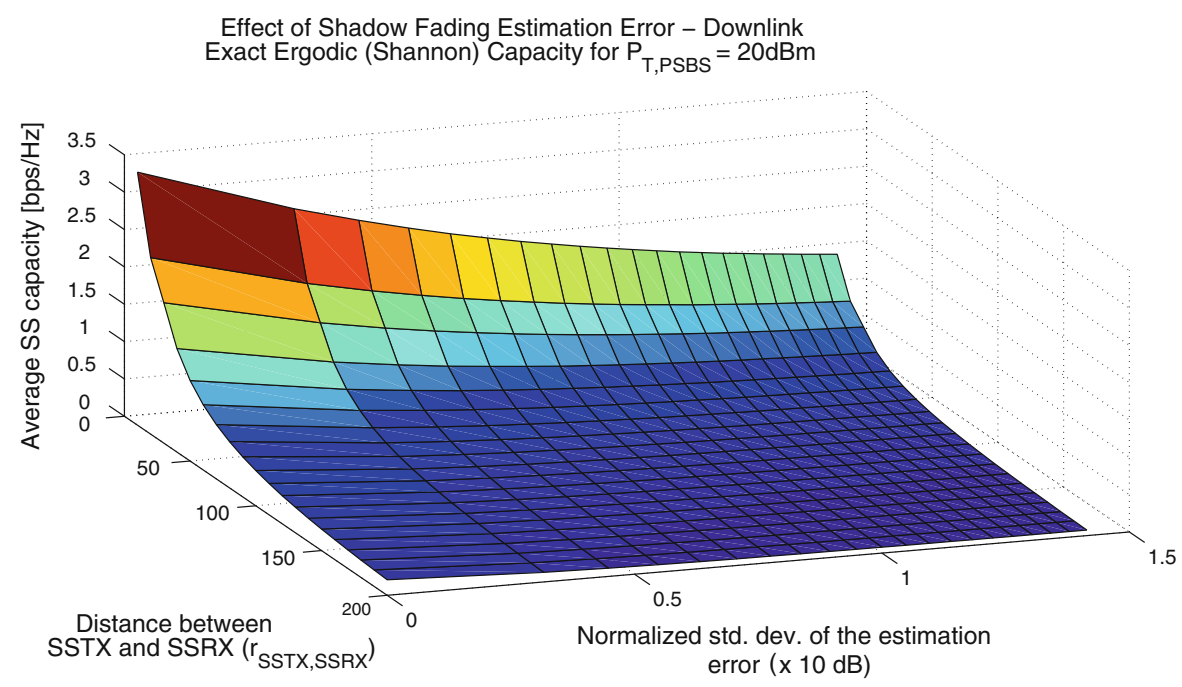

(a) Ergodic Capacity of SS

Effect of Shadow Fading Estimation Error - Downlink Average SINR Loss at PSRX for $\mathrm{P}_{\mathrm{T}, \mathrm{PSBS}}=20 \mathrm{dBm}$

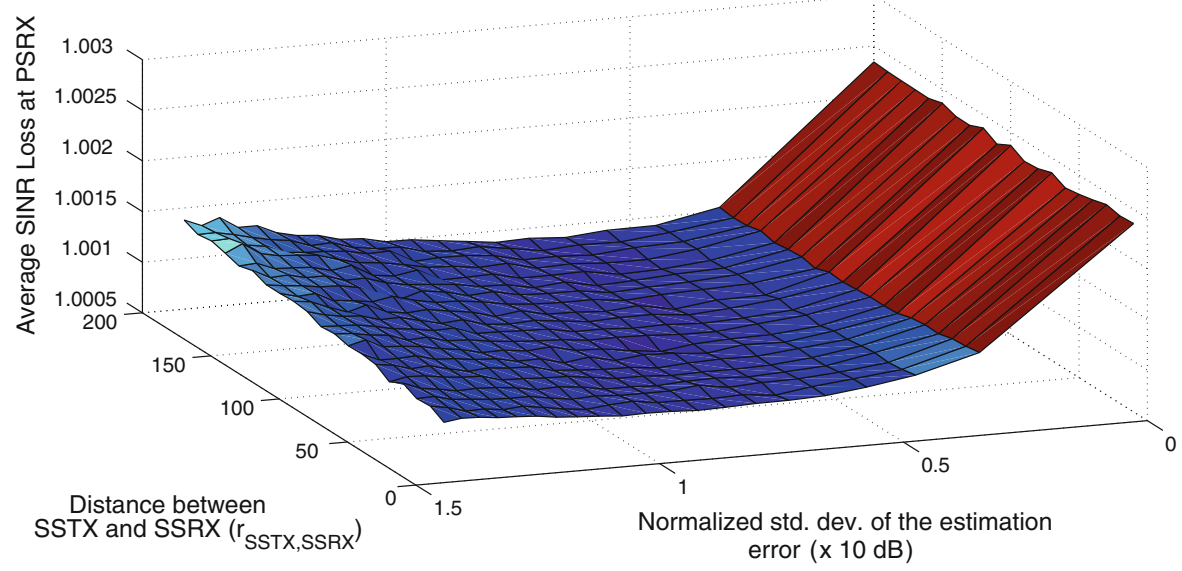

(b) Average SINR Loss at PS

Fig. 4 Effects of shadow fading estimation error on a the ergodic capacity of the link between SSTX and SSRX, b average SINR loss at the PSRX versus normalized standard deviation of the estimation error and the distance between SSTX and SSRX for downlink communications

$q \in\{0,1\}$ corresponding to the cases of noise-free shadow fading estimation and estimation under noise with $10 \mathrm{~dB}$ variance, respectively. These are depicted in Fig. 5a, b.

\subsection{Effect of Average SINR Loss Constraint at the PS receiver}

In Sect. 5.3, we have employed a strict constraint on the average SINR loss, namely $\mathrm{SINR}_{\mathrm{p}, \text { loss }}=0.01 \mathrm{~dB}$. In this part, we repeat the analysis in the previous section by relaxing the constraint on the average SINR loss at the primary system receiver. The exact ergodic 


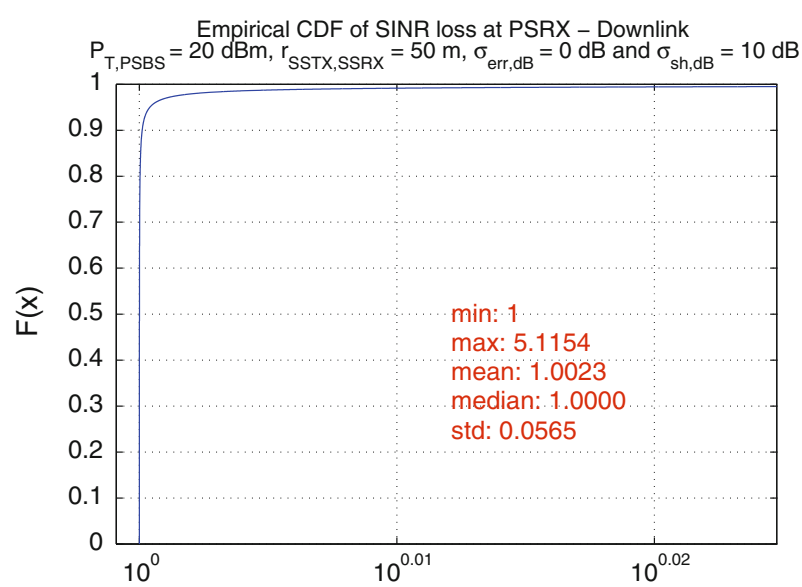

(a) Noise-free SINR Loss - Downlink

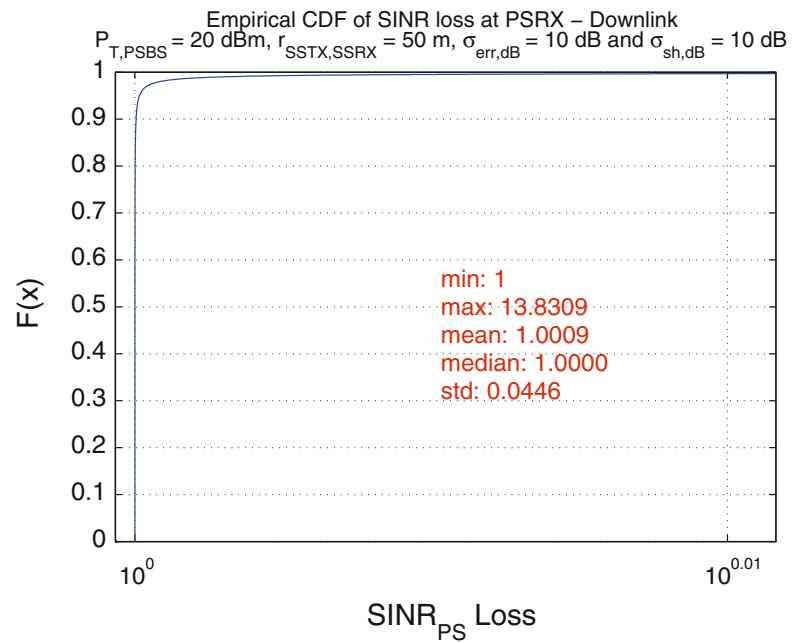

(b) Noisy SINR Loss - Downlink

Fig. 5 Effects of shadow fading estimation error on the empirical cumulative distribution function of the SINR loss at $r_{s s}=50 \mathrm{~m}$ : a noiseless scenario, b under noise with $10 \mathrm{~dB}$ variance for downlink communications

capacity performance of the proposed power adaptation strategy under the relaxed SINR loss constraint of $1 \mathrm{~dB}$ is presented for both noiseless and noisy measurement cases in Figs. 6, 7a, b.

\section{Conclusion}

A novel power adaptation strategy has been proposed to maximize the ergodic capacity of the secondary system subject to an average SINR loss constraint at the primary system for a single-cell network. The closed form water-filling solution can operate in the absence of any path loss estimates depending solely on the instantaneous value of the shadow gain between secondary transmitter and primary receiver. Numerical simulations have been provided to 


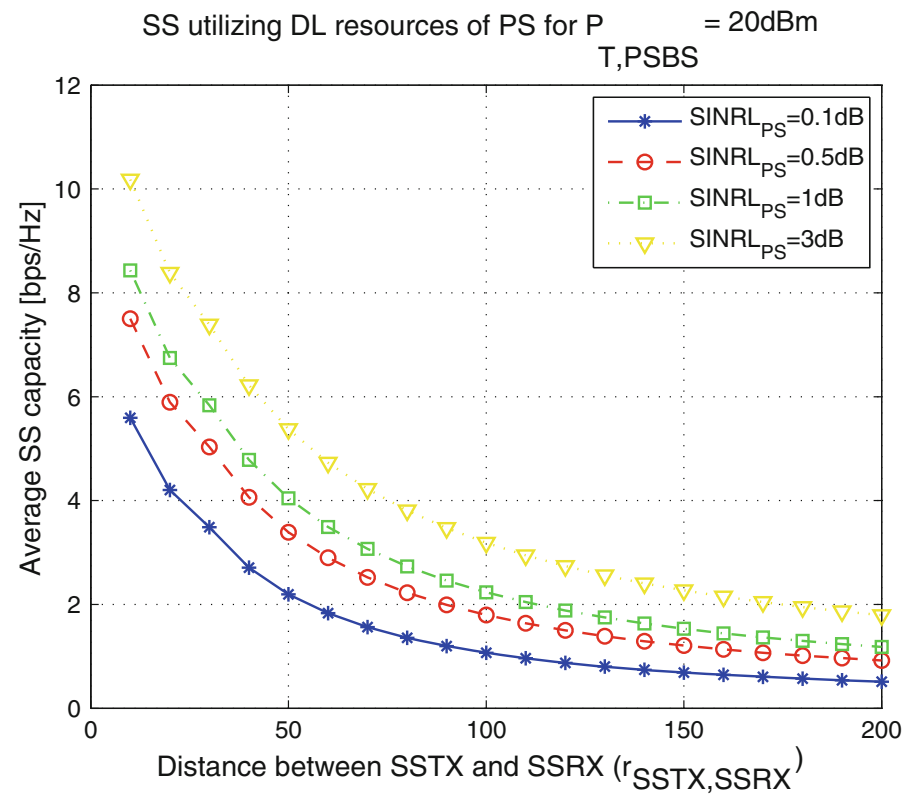

Fig. 6 Exact ergodic capacity versus distance between SSTX and SSRX under various average SINR loss constraints using the proposed power adaptation strategy for downlink communications

corroborate the theoretical results. More explicitly, we have provided plots for the exact ergodic capacity of the proposed strategy and discussed the effect of the shadow fading exponent's variance on the performance. By employing uniformly distributed estimation errors for the shadow fading on the link between SS transmitter and PS receiver, we have analyzed the effects on the ergodic capacity of the SS and the probabilistic structure of the SINR loss at the PS receiver. Furthermore, we have shown how the proposed strategy behaves as the average SINR loss constraint is relaxed.

\section{Appendix}

Derivation of Instantaneous SINR Loss at PS receiver

When no SS user is present in the PS service area, the SNR at the PS receiver can be written as

$$
\mathrm{SNR}_{p}=\frac{P_{p} G\left(r_{p p}, \xi_{p p}\right)}{N_{p}}
$$

where $P_{p}$ is the transmission power of the PS, $G\left(r_{p p}, \xi_{p p}\right)$ is the combined shadowing and path gain between PS transmitter and receiver, and $N_{p}$ is the noise power at the PS receiver. When an SS transmitter is present and interferes with the PS receiver, the SINR at the PS receiver can be written as

$$
\operatorname{SINR}_{p}=\frac{P_{p} G\left(r_{p p}, \xi_{p p}\right)}{P_{s}(\mathbf{r}, \xi) G\left(r_{s p}, \xi_{s p}\right)+N_{p}}
$$




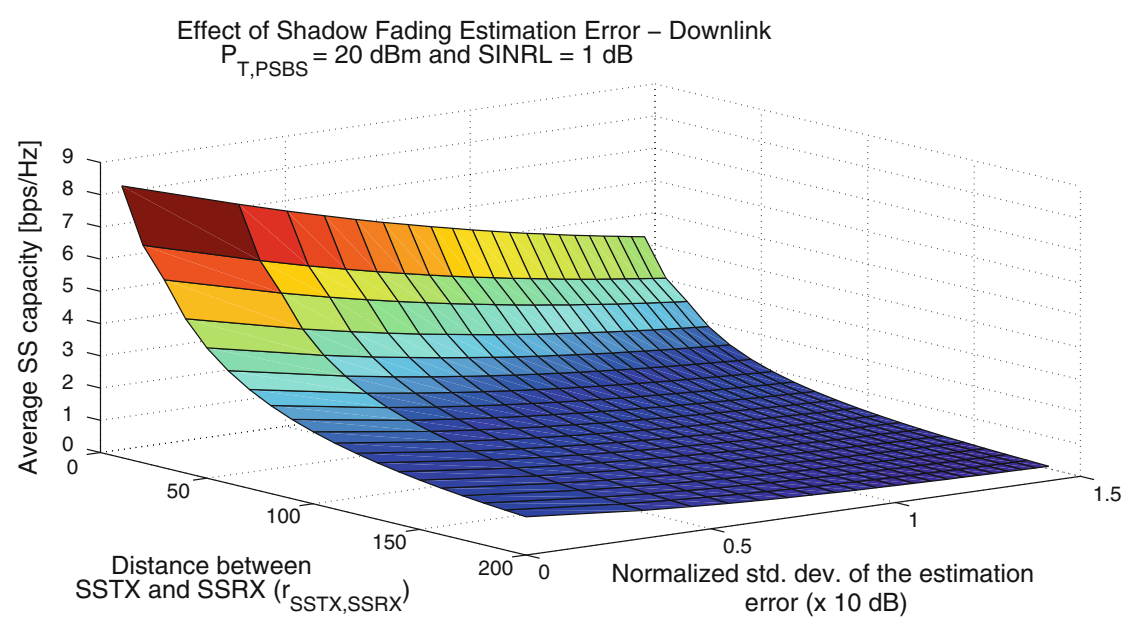

(a) Ergodic Capacity of SS

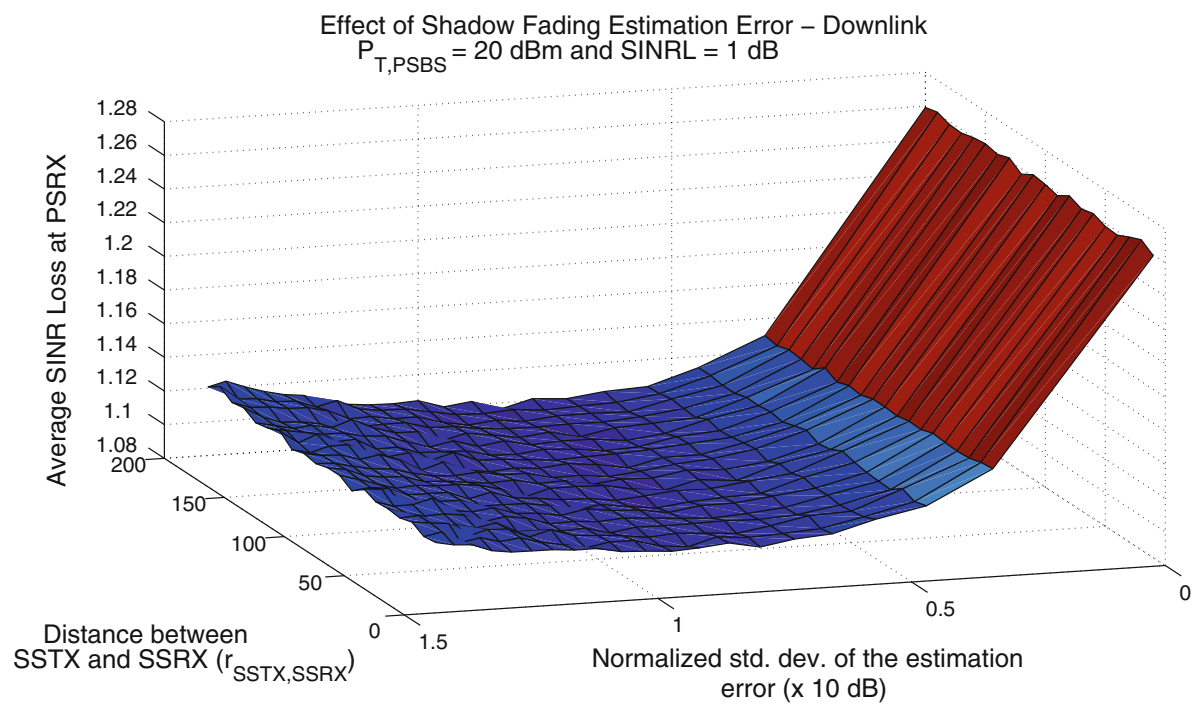

(b) Average SINR Loss at PS

Fig. 7 Effects of shadow fading estimation error on a the ergodic capacity of the link between SSTX and SSRX, b SINR loss at the PSRX versus normalized standard deviation of the estimation error and the distance between SSTX and SSRX for desired average SINR $_{\mathrm{p}, \text { loss }}=1 \mathrm{~dB}$ in the case of downlink communications

where $P_{s}(\mathbf{r}, \boldsymbol{\xi})$ is the transmission power of the SS and $G\left(r_{s p}, \xi_{s p}\right)$ is the combined shadowing and path gain between SS transmitter and PS receiver. From (33) and (34), the instantaneous SINR loss at the PS receiver due to the SS transmission is given as

$$
\mathrm{SINR}_{\mathrm{p}, \text { loss }}=\frac{\mathrm{SNR}_{p}}{\mathrm{SINR}_{p}}=1+\frac{P_{s}(\mathbf{r}, \boldsymbol{\xi}) G\left(r_{s p}, \xi_{s p}\right)}{N_{p}} .
$$

Equation (35) indicates that $\mathrm{SINR}_{\mathrm{p}, \text { loss }}$ depends on the interference level from the SS and the thermal noise at the PS victim, and it is independent of the PS tranmit power. 


\section{References}

1. Federal Communications Commission. (Nov. 2002). Spectrum policy task force. ET Docket No. 02-135, Technical Report.

2. Mitola, III, J., \& Maguire, G.Q. (1999). Cognitive radio: Making software radios more personal. IEEE Personal Communications, 6(4) 13-18.

3. Haykin, S. (2005). Cognitive radio: Brain-empowered wireless communications. IEEE Journal on, Selected Areas in Communications, 23(2), 201-220.

4. Gastpar, M. (2007). On capacity under receive and spatial spectrum-sharing constraints. IEEE Transactions on, Information Theory, 53(2), 471-487.

5. Ghasemi, A., \& Sousa, E. (2007). Fundamental limits of spectrum-sharing in fading environments. IEEE Transactions on Wireless Communications, 6(2), 649-658.

6. Goldsmith, A. J., \& Varaiya, P. P. (1997). Capacity of fading channels with channel side information. IEEE Transactions on Information Theory, 43(6), 1986-1992.

7. Biglieri, E., Proakis, J., \& Shamai, S. (1998). Fading channels: Information-theoretic and communications aspects. IEEE Transactions on Information Theory, 44(6), 2619-2692.

8. Liang, Y.-C., Zhang, R., \& Cioffi, J. (2006). Subchannel grouping and statistical waterfilling for vector block-fading channels. IEEE Transactions on Communications, 54(6), 1131-1142.

9. Musavian, L., \& Aissa, S. (2007). Ergodic and outage capacities of spectrum-sharing systems in fading channels. In Proceedings of the IEEE global telecommunication conference (GLOBECOM'07), pp. 33273331.

10. Kang, X., Liang, Y., Nallanathan, A., Krishna, H., \& Zhang, R. (2009). Optimal power allocation for fading channels in cognitive radio networks: Ergodic capacity and outage capacity. IEEE Transactions on Wireless Communications, 8(2), 940-950.

11. Zhang, R., \& Liang, Y.-C. (2008). Exploiting multi-antennas for opportunistic spectrum sharing in cognitive radio networks. IEEE Journal of Topics in Signal Processing, 2(1), 88-102.

12. Zhang, L., Liang, Y.-C., \& Xin, Y. (2008). Joint beamforming and power allocation for multiple access channels in cognitive radio networks. IEEE Journal on Selected Areas in Communications, 26(1), 38-51.

13. Cho, H., \& Andrews, J. (2009). Upper bound on the capacity of cognitive radio without cooperation. IEEE Transactions on Wireless Communications, 8(9), 4380-4385.

14. Vu, M., Devroye, N., Sharif, M., \& Tarokh, V. (2007). Scaling laws of cognitive networks. In Proceedings of the international conference cognitive radio oriented wireless networks and communications (Crowncom), pp. 2-8.

15. Musavian, L., \& Aissa S. (2008). Capacity of spectrum-sharing channels with minimum-rate requirements. In Proceedings of the IEEE international conference communications (ICC), pp. 4639-4643.

16. Chen, Y., Yu, G., Zhang, Z., Chen, H. H., \& Qiu, P. (2008). On cognitive radio networks with opportunistic power control strategies in fading channels. IEEE Transactions on Wireless Communications, 7(7), 27522761.

17. Kang, X., Zhang, R., Liang, Y.-C., \& Garg, H. K. (2011). Optimal power allocation strategies for fading cognitive radio channels with primary user outage constraint. IEEE Journal on Selected Areas in Communications, 29(2), 374-383.

18. Gong, X., Vorobyov, S., \& Tellambura, C. (2011). Optimal bandwidth and power allocation for sum ergodic capacity under fading channels in cognitive radio networks. IEEE Transaction on Signal Processing, 59(4), 1814-1826.

19. Goldsmith, A. (2009). Wireless communications. Cambridge: Cambridge University Press.

20. ITU-R Recommendation M.1225. (1997). Guidelines for evaluation of radio transmission technologies for imt-2000.

21. IEEE P802.11 Wireless LANs. (2004). TGn channel models. IEEE 802.11-03/940r4.

22. Boyd, S., \& Vandenberghe, L. (2004). Convex optimization. Cambridge: Cambridge University Press.

23. Algans, A., Pedersen, K. I., \& Mogensen, P. E. (2002). Experimental analysis of the joint statistical properties of azimuth spread, delay spread, and shadow fading. IEEE Journal on Selected Areas in Communications, 20(3), 523-531.

24. Jalden, N., Zetterberg, P., Ottersten, B., \& Garcia, L. (2007). Inter- and intrasite correlations of large-scale parameters from macrocellular measurements at $1800 \mathrm{mhz}$. EURASIP Journal on Wireless Communications and Networking, 2007, 12.

25. Rappaport, T. (1996). Wireless communications: Principles and practice. Upper Saddle River, NJ: Prentice-Hall Inc.

26. Hata, M. (1980). Empirical formula for propagation loss in land mobile radio service. IEEE Transactions on Vehicular Technology, 29, 317-325.

27. Poor, H. V. (1994). An introduction to signal detection and estimation. New York: Springer. 
28. Stber, G. (2001). Principles of mobile communication (2nd ed.). Norwell, MA: Kluwer, Prentice-Hall Inc.

29. Tepedelenlioglu, C., Abdi, A., Giannakis, G. B., \& Kaveh, M. (2001). Estimation of doppler spread and signal strength in mobile communications with applications to handoff and adaptive transmission. Wireless Communications and Mobile Computing, 1(2), 221-242.

30. Wong, D., \& Cox, D. C. (1999). Estimating local mean signal power level in a rayleigh fading environment. IEEE Transactions on Vehicular Technology, 48(3), 956-959.

31. Goldsmith, A. J., Greenstein, L. J., \& Foschini, G. J. (1994). Error statistics of real-time power measurements in cellular channels with multipath and shadowing. IEEE Transactions on Vehicular Technology, 43(3), 439-446.

32. Ko, Y. C., \& Alouini, M. S. (2003). Estimation of nakagami-m fading channel parameters with application to optimized transmitter diversity systems. IEEE Transactions on Wireless Communications, 2(2), 250259.

33. Jiang, T., Sidiropoulos, N. D., \& Giannakis, G. B. (2003). Kalman filtering for power estimation in mobile communications. IEEE Transactions on Wireless Communications, 2(1), 151-161.

34. Dogandzic, A., \& Zhang, B. (2005). Dynamic shadow-power estimation for wireless communications. IEEE Transactions on Signal Processing, 53(8), 2942-2948.

35. Fishman, G.S. (1996). Monte Carlo: Concepts, algorithms, and applications. Springer Series in Operations Research. New York: Springer.

\section{Author Biographies}

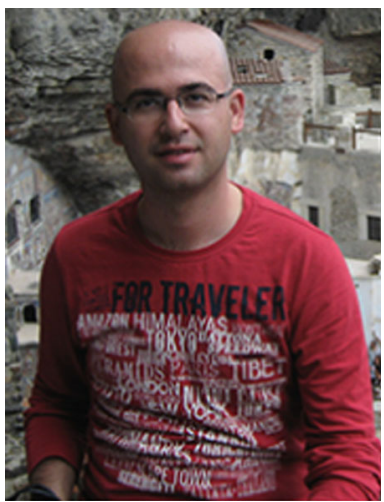

Berkan Dulek received the B.S., M.S. and Ph.D. degrees in electrical and electronics engineering from Bilkent University in 2003, 2006 and 2012, respectively. From 2007 to 2010, he worked at Tubitak Bilgem Iltaren Research and Development Group. From 2012 to 2013, he was a postdoctoral research associate at the Department of Electrical Engineering and Computer Science, Syracuse University, Syracuse, NY. His research interests are in statistical signal processing, detection and estimation theory, and wireless communications.

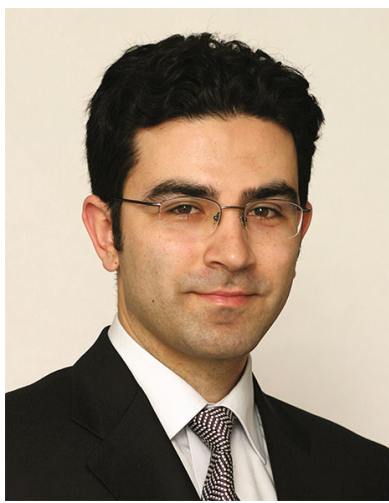

Sinan Gezici received the B.S. degree from Bilkent University, Turkey in 2001, and the Ph.D. degree in electrical engineering from Princeton University in 2006. From 2006 to 2007, he worked at Mitsubishi Electric Research Laboratories, Cambridge, MA. Since 2007, he has been with the Department of Electrical and Electronics Engineering at Bilkent University, where he is currently an Associate Professor. Dr. Gezici's research interests are in the areas of detection and estimation theory, wireless communications, and localization systems. Among his publications in these areas is the book Ultra-wideband Positioning Systems: Theoretical Limits, Ranging Algorithms, and Protocols (Cambridge University Press, 2008). Dr. Gezici is an associate editor for IEEE TRANSACTIONS ON COMMUNICATIONS, IEEE WIRELESS COMMUNICATIONS LETTERS, and the Journal of Communications and Networks. 

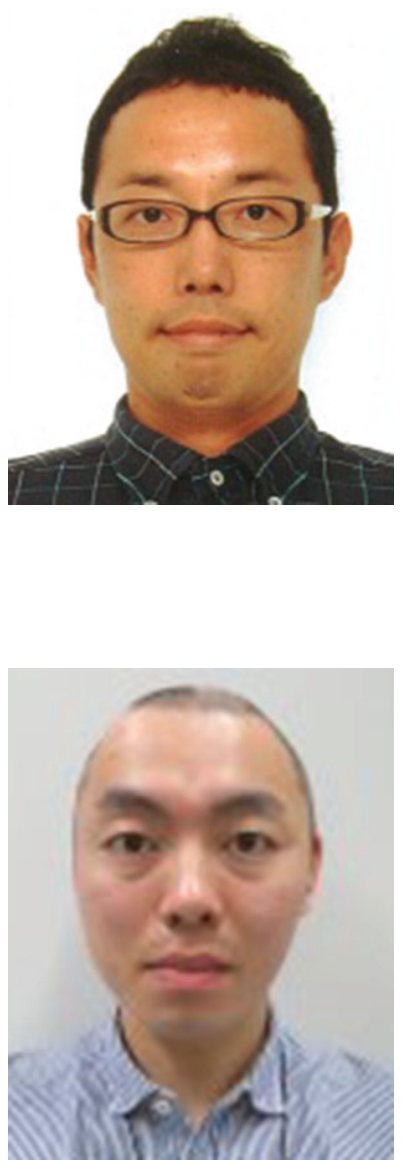

Ryo Sawai received the B.E., M.E. and Ph.D. degrees in electrical and electronic engineering from Chuo University, Tokyo, Japan, in 1998, 2000 and 2002, respectively. From July 1999 to September 1999, he joined Nokia student exchange program in Helsinki, Finland. From October 1999 to May 2002, he was a student trainee with National Institute of Information and Communications Technology, Yokosuka, Japan. He is currently a senior researcher at Sony Corporation, Japan, and was also student of an executive Management of Technology (MOT) course at CICOM-ISL (Innovation Strategy and Leadership basic program) from January 2012 to September 2013. His research interests include advanced coding design, e.g. Turbo code/decoder and low density parity check code/decoder, reconfigurable hardware architecture design, multi-antenna processing technologies, cognitive radio, multi/many core based processing technology and cloud architecture design. He has been contributing to IEEE 1900.6 standard, IEEE 802.19.1 and CEPT SE43. He received IEEE VTS Japan Researcher's Encouragement Award in 2001.

Ryota Kimura earned his B.E. and M.E. degrees from Chuo University, Japan, in 2003 and 2005, respectively, and earned his Ph.D. degree from Waseda University, Japan, in 2008. From 2002 to 2008, he was also a research trainee at National Institute of Information and Communications Technology (NICT). Currently he is a research engineer at Sony Corporation. His research interests include cognitive radio, TV white spaces, LTE/LTE-A and future mobile communication systems. $\mathrm{He}$ is a member of IEEE and that of IEICE Japan. 